\title{
Prolonged maternal vitamin C deficiency overrides preferential fetal ascorbate transport but does not influence perinatal survival in guinea pigs
}

\author{
Janne G. Schjoldager, Pernille Tveden-Nyborg and Jens Lykkesfeldt* \\ Department of Veterinary Disease Biology, Faculty of Health and Medical Sciences, University of Copenhagen, \\ 9 Ridebanevej, DK-1870 Frederiksberg C, Denmark \\ (Submitted 4 October 2012 - Final revision received 24 January 2013 - Accepted 24 January 2013 - First published online 16 April 2013 )
}

\section{Abstract}

Human and guinea pig fetuses are completely dependent on an adequate maternal vitamin C (vitC) intake. Shortage of micronutrients can have negative implications for fetal health and pregnancy outcome; however, knowledge of maternal vitC deficiency's impact on fetal development is sparse and reports of pregnancy outcome have been divergent. The present study investigated whether maternal vitC deficiency affects pregnancy outcome and plasma vitC distribution between the mother and the offspring in a guinea pig model. A total of eighty pregnant Dunkin Hartley guinea pigs were randomised into two weight-stratified groups receiving either a deficient $(100 \mathrm{mg} / \mathrm{kg}$ DEF) or a control $(923 \mathrm{mg} / \mathrm{kg}$ CTRL) diet. VitC levels were measured in plasma during pregnancy and postpartum, and in the plasma and brain of newborns. Pregnancy outcome was recorded with respect to birth weight and perinatal survival and were similar between groups. Plasma vitC in dams declined throughout gestation in both groups $(P<0 \cdot 01)$. Compared with maternal plasma vitC, plasma vitC of newborn pups was found to be significantly lower in the DEF group $(P<0 \cdot 001)$ and higher in the CTRL group $(P<0 \cdot 001)$, respectively. Brain vitC levels were significantly reduced in DEF newborn pups $(P<0 \cdot 001)$. The present results indicate that preferential transport of vitC from the mother to the fetus is overridden during sustained maternal vitC deficiency, maintaining maternal vitC concentration at the expense of the offspring. This contradicts the notion that a fetus is protected from vitC deficiency by the placental Na-dependent vitC co-transporter, SVCT2, thus fetal development may be susceptible to the negative effects of maternal vitC deficiency.

Key words: Vitamin C: Deficiency: Fetuses: Pregnancy outcomes

Malnutrition during fetal development and infancy - e.g. DHA, Fe and folic acid deficiencies ${ }^{(1-5)}$ - can have severe negative implications for the brain and nervous system and may also affect the outcome of pregnancy, leading to premature births and the birth of weak or stillborn offspring ${ }^{(6,7)}$. We have hypothesised that vitamin $\mathrm{C}$ (vitC) is a pivotal factor in normal brain development ${ }^{(8)}$, and have recently reported that prenatal vitC deficiency leads to persistent postnatal hippocampal volume reduction and impaired neuronal migration ${ }^{(9)}$. Moreover, we have shown that postnatal vitC deficiency has detrimental effects on hippocampal neuron number and function $^{(10)}$, and that severe postnatal vitC depletion in weanling guinea pigs leads to oxidative stress in the brain ${ }^{(11)}$. In mice, a functional vitC transport (mediated by the Na-dependent vitC co-transporter 2; SVCT2) has been shown to be essential for perinatal survival ${ }^{(12)}$. However, knowledge of vitC deficiency's impact on in utero fetal development remains sparse.

Due to a mutation in the gene encoding for L-gulonolactone oxidase required for ascorbate synthesis, humans and guinea pigs are dependent on a dietary vitC source ${ }^{(13)}$. Consequently, fetal supply of vitC relies on an adequate maternal dietary intake $^{(14)}$ and, expectedly, the maternal:fetal transport ratio has been shown to be dependent on maternal vitC status $^{(15-17)}$. In humans, vitC in plasma shows a declining maternal plasma concentration during pregnancy ${ }^{(15)}$, while vitC levels in newborns are typically higher than those in their post-parturient mothers ${ }^{(16,17)}$. This is most probably due to an active placental transport of ascorbate as indicated in both in vitro and in vivo studies ${ }^{(12,18,19)}$. As with humans, the guinea pig has a haemochorial placenta and is a validated animal model for placental transfer ${ }^{(20-22)}$. This makes the guinea pig a rational model for studying the putative effects of dietary-imposed chronic vitC deficiency on pregnancy outcome.

In humans, a daily intake of $200-400 \mathrm{mg}$ vitC for adults results in plasma concentrations of about $70 \mu \mathrm{mol} / \mathrm{l}^{(23,24)}$, at which point tissue saturation is achieved. However, vitC deficiency (hypovitaminosis C) defined as a plasma concentration $<23 \mu \mathrm{mol} / \mathrm{l}$ and severe vitC deficiency (a plasma concentration $<11 \mu \mathrm{mol} / 1)^{(25)}$ remains surprisingly common.

Abbreviations: CTRL, control group; DEF, deficient group; SVCT2, sodium-dependent vitamin C co-transporter 2; vitC, vitamin C.

*Corresponding author: Professor J. Lykkesfeldt, fax +45353 53514, email jopl@sund.ku.dk 
Even in the developed world, large cross-sectional studies have found that vitC deficiency affects between 7 and $40 \%$ of the population, with high-risk subpopulations such as pregnant women, children and smokers being most vulnerable $^{(17,26-28)}$. In spite of this high prevalence of vitC deficiency, its potential impact on pregnancy and newborns has only been the subject of little attention.

Using guinea pigs as an in vivo model, the present study investigated the impact of a prolonged subclinical maternal vitC deficiency during the second and third trimesters on maternal and early postnatal vitC status, birth weight and perinatal survival in guinea pig offspring.

\section{Materials and methods}

\section{In vivo study}

The study was approved by the Danish Animal Experimentation Inspectorate. Upon arrival, a total of eighty timemated Dunkin Hartley guinea pigs (Charles River Laboratories), second-time breeders at gestational day 18, were weighed and tagged with a $12 \mathrm{~mm}$ microchip subcutaneously in the neck for identification (Pet-Id; Danworth Farm). The animals were randomly assigned to one of two weight-stratified groups that received special quality-controlled diets (Special Diets Services) either sufficient in vitC ( $923 \mathrm{mg} / \mathrm{kg}$ by analysis; product code: $829427-\mathrm{B} \# 32786$; control (CTRL) group, $n$ 30) or deficient in vitC $(100 \mathrm{mg} / \mathrm{kg}$ by analysis; product code: 829415-B\#32785; deficient (DEF) group, $n 50$ ). The diets were produced to meet the nutritional requirements of guinea pigs and were identical in all aspects except vitC content. We have previously shown that a dose of $100 \mathrm{mg} v i t C / \mathrm{kg}$ induces a non-scorbutic vitC deficiency in guinea pigs ${ }^{(29,30)}$. Accordingly, none of the animals exhibited any clinical signs of scurvy during the study. The groups were allowed ad libitum feeding, dried hay (tested negative for vitC content) and drinking water. They were housed in large floor pens with a $12 \mathrm{~h}$ light cycle and tended several times daily by trained staff. All newborn pups (live: $n$ 285, dead: $n$ 58) were recorded within a maximum of $24 \mathrm{~h}$ from birth and viable pups were weighed, marked for identification and their sex determined.

Dams and pups were weighed once and twice weekly, respectively, and eight dams from each group (each time randomly chosen by a computer) were assigned for the collection of blood samples at arrival (ultimo first trimester, gestational day 18) and at the beginning of the third trimester (gestational day 40). Blood samples were collected from the saphenous vein on its superficial course on the tibia $(300 \mu \mathrm{l})$. Only one pregnant female from the CTRL group died due to pregnancy toxaemia and five animals proved not to have conceived.

\section{Euthanasia}

At postnatal days 2-7 (pups) and postpartum days 7-10 (dams), four groups of newborn pups ( $n$ 10-11; females/ males; CTRL/DEF) and two groups of dams ( $n$ 5-10; CTRL/DEF) were anaesthetised with inhalation of isoflurane
(Isoba Vet 100\%; Intervet International), and blood ( $4 \mathrm{ml}$ ) was gently collected by cardiac puncture with an 18G hypodermic needle in a $5 \mathrm{ml}$ syringe previously flushed with $15 \%$ tripotassium-EDTA ${ }^{(31)}$. Subsequently, while still anaesthetised, the animals were euthanised by exsanguination and decapitation. Plasma from dams and female/male pups and brains from female pups (either the right or left hemisphere, randomly assigned by a computer and divided by section through the cerebral longitudinal fissure) were immediately frozen at $-80^{\circ} \mathrm{C}$ until biochemical analyses were performed. The remaining brain tissues, pups and postpartum dams were allocated to other studies.

\section{Biochemical analyses}

Analysis of ascorbate and dehydroascorbic acid in a metaphosphoric acid-stabilised plasma and brain homogenate was performed at HPLC with coulometric detection as described previously $^{(32,33)}$. As a measure of ascorbate oxidation, the ascorbate oxidation ratio in the brain was calculated as a percentage of dehydroascorbic acid of total vitC. Glutathione was measured spectrofluorometrically according to the method by Hissin \& Hilf $^{(34)}$ modified for microtitre plates. Lipid oxidation was assessed by measuring malondialdehyde as described previously ${ }^{(35)}$ and $8-\mathrm{F}_{2}$-isoprostanes as described by the assay kit manufacturer (catalogue no. 516351; Cayman Chemicals).

\section{Statistical analysis}

Differences in body weight and plasma concentrations of ascorbic acid were evaluated by one-way ANOVA followed by Tukey's multiple comparison test to describe differences in means. In the case of a non-Gaussian distribution as established by the D'Agostino and Pearson omnibus normality test, evaluation was conducted by the Kruskal-Wallis test followed by Dunn's multiple comparisons. Data were analysed using Statistica (Statsoft). To assess the differences in perinatal mortality, logistic regression analysis corrected for overdispersion was carried out in SAS version 9.2 (SAS Institute, Inc.). The significance level for all statistical analyses was $P<0.05$ and data are expressed as means and standard deviations unless otherwise stated.

\section{Results}

\section{Maternal weight gain and birth weight}

No signs of pregnancy-associated disease or dystocia were recorded for dams brought to term. Maternal weight gain during gestation was similar between the groups (Fig. 1). Due to group housing in large pens, individual litter sizes could not be recorded and because of a few incidences of cannibalism, the recorded number of perinatally dead pups could be slightly flawed. There was no significant difference in the birth weight of live-born pups between the groups (CTRL 80.0 (SD 18.1)g $v$. DEF 80.7 (SD 15.9) g) or sex (females 80.06 (SD 16.59) g $v$. males 80.97 (SD 16.91) g). 


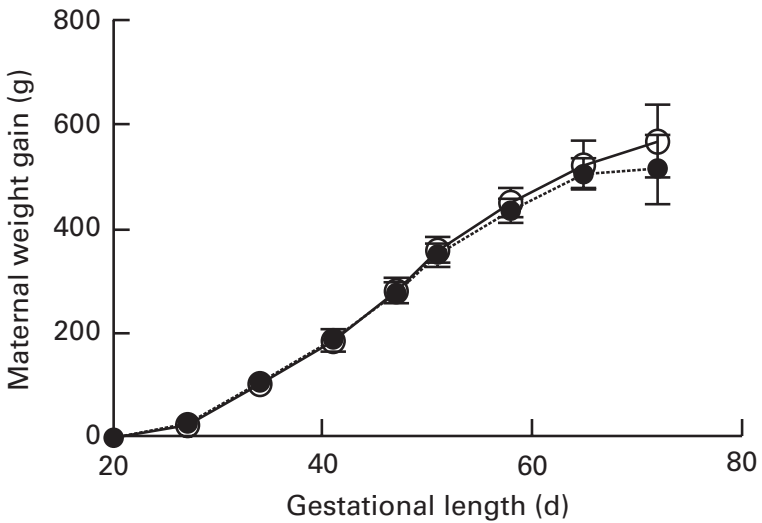

Fig. 1. Maternal weight gain during gestation. Body weights were recorded once weekly. Data are shown as weight gain normalised to body weight on arrival. Data are presented using body-weight gestational day 18 as baseline. Values are means, with their standard errors represented by vertical bars. There was no significant difference between the deficient $(\theta, n 45)$ and control (-., $n$ 29) dams at any of the recorded time points.

\section{Plasma levels of total vitamin C concentration}

Upon arrival at gestational day 18, dams displayed plasma levels of total vitC of 39.8 (SD 11.8) $\mu \mathrm{mol} / \mathrm{l}$. As expected, the dietary regimen was reflected in the plasma levels of total vitC, CTRL dams had significantly higher plasma vitC levels than DEF dams at both gestational day 40 (CTRL: $36 \cdot 3$ (SD 6.8) $v$. DEF: 3.9 (SD 1.2) $\mu \mathrm{mol} / \mathrm{l}$ ) and at postpartum days 7-10 (CTRL: $21 \cdot 8$ (SD 9.0) $v$. DEF: $3 \cdot 1$ (SD $2 \cdot 0) \mu \mathrm{mol} / 1$ ), as displayed in Fig. 2. Towards parturition, vitC status in the CTRL dams declined significantly $(P<0.01 ;$ Fig. 2). In the DEF dams, plasma vitC levels declined significantly within 3 weeks after arrival (to 3.9 (SD 1.2$) \mu \mathrm{mol} / \mathrm{l}$ ) and remained at this low level during pregnancy and parturition $(P<0.001$; Fig. 2). The dietary regimen was also reflected in plasma vitC concentrations of newborn pups. However, as no effect of sex on vitC concentration could be detected in either group of newborns, vitC data from both sexes were pooled within the groups. The newborn CTRL pups had significantly higher plasma vitC concentrations (CTRL pups: $66 \cdot 3$ (SD 27.4) $\mu \mathrm{mol} / \mathrm{l}$ ) compared with the CTRL dams at parturition $(P<0.001$; Fig. 3(a)); however, in contrast, the newborn DEF pups had a significantly lower plasma level of vitC (1.6 (sD 0.7$) \mu \mathrm{mol} / \mathrm{l})$ compared with their mothers $(P<0 \cdot 001 ;$ Fig. 3(b)).

\section{Oxidative stress markers in the brain}

In the newborn DEF female pups, vitC concentrations in the brain decreased by $60 \%$ as a result of the maternal dietary regimen $(P<0 \cdot 001$; Table 1$)$. No effects on the measured oxidative stress and damage markers, i.e. ascorbate oxidation ratio, glutathione, malondialdehyde or $8-\mathrm{F}_{2}$-isoprostanes, were found in the brain (Table 1).

\section{Perinatal survival}

A total of 143 CTRL ( $n 29$ dams) and 200 DEF ( $n 45$ dams) pups were delivered. Of these, eighteen CTRL and forty DEF pups were recorded as dead perinatally (i.e. dead in utero or within a maximum of $24 \mathrm{~h}$ after birth). All dead pups appeared to be delivered at or very close to term. No macroscopic causes of death could be identified, except from one mummified fetus (DEF) and five moribund newborns (DEF) that were immediately euthanised by cervical dislocation. To evaluate the association between the maternal diet and the offspring's risk of perinatal death, data were fitted to a logistic regression model corrected for overdispersion between day and diet. When corrected for overdispersion, the effect of diet was not significant $(P=0 \cdot 18)$ with an OR for perinatal death being (DEF $v$. CTRL) 1.7 (95\% CI 0.8, - 3.9).

\section{Discussion}

In both humans and guinea pigs, maternal vitC intake affects vitC concentrations in offspring ${ }^{(36-39)}$. During gestation, maternal plasma levels decline even when maternal vitC intake is high ${ }^{(15)}$. In agreement, the present study also reports of a declining maternal vitC concentration in both investigated dietary groups during gestation. Interestingly, DEF pups were significantly lower in vitC than DEF dams at gestational day 40 , i.e. during the third trimester.

It has been proposed that the placental-fetal consolidation is genetically programmed to alter maternal physiology to benefit the fetus and to ensure survival until birth, sometimes at the expense of the mother ${ }^{(40)}$. Conditions such as gestational hypertension and pre-eclampsia have been suggested to develop when the mother is unable to meet fetal nutritional requirements ${ }^{(40)}$ and in cases of insufficient $\mathrm{Ca}$, fetal supply is ensured by maternal release from the bone, ultimately decreasing maternal skeletal strength ${ }^{(41)}$. In humans, an insufficient maternal vitC intake, defined from maternal blood samples taken at parturition or dietary information provided

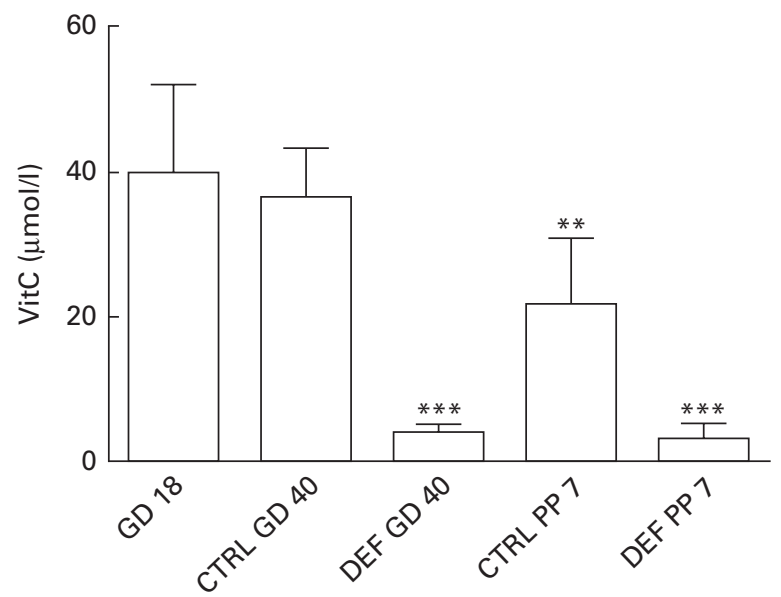

Fig. 2. Plasma vitamin $C$ (vitC) levels in dams. Initial vitC levels were recorded upon arrival at gestational day (GD) 18. On both GD 40 and postpartum day (PP) 7, plasma vitC levels in the deficient (DEF) dams were significantly lower compared with the control (CTRL) dams $\left({ }^{* *} P<0.001\right)$, reflecting the two applied dietary regimens. Also, plasma vitC levels in the CTRL dams declined significantly during gestation $\left({ }^{\star *} P<0.01\right)$. Data are obtained from randomly chosen pregnant/postpartum dams (n 5-10; CTRL/DEF) at each time point. Values are means, with standard deviations represented by vertical bars. 

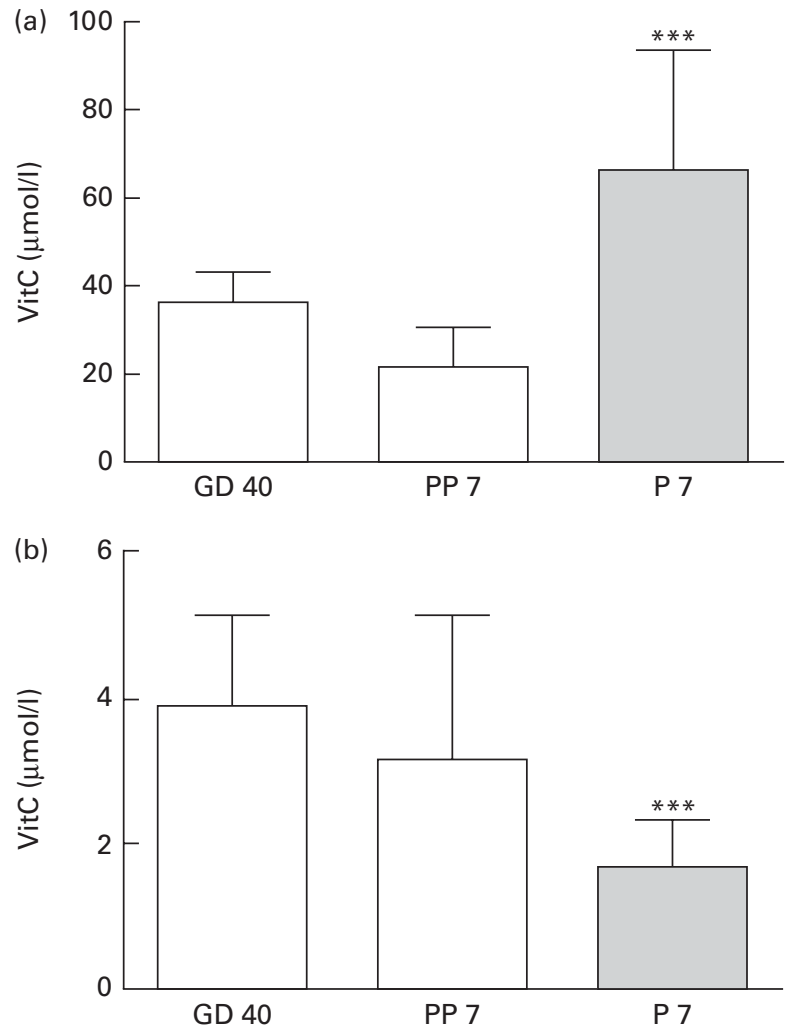

Fig. 3. Plasma vitamin $C$ (vitC) levels in dams and offspring. (a) Newborn control (CTRL) female ( $n$ 10) and male $(n 10)$ pups at postnatal day (P) 7 had significantly higher vitC levels than the CTRL dams at postpartum day(PP) $7\left(^{* \star *} P<0.001\right)$. P7 female and male data are pooled as no effect of sex was observed. (b) Newborn deficient (DEF) female ( $n$ 11) and male $(n$ 10) pups at postnatal day $(P) 7$ had significantly lower plasma vitC than the DEF dams during gestation ( $\left.{ }^{\star \star \star} P<0.001\right)$. P7 female and male data are pooled as no effect of sex was observed. Values are means, with standard deviations represented by vertical bars.

by pregnant women, has been reported to increase fetal: maternal plasma vitC ratios (i.e. fetal levels increase and maternal levels decrease) even under conditions associated with maternal deficiency ${ }^{(15,42)}$. This has led to the proposal of a selective retention of vitC by the placenta, ensuring adequate fetal levels even during severe maternal deficiency. However, it should be noted that the cited reports on maternal vitC deficiency date back to the late 1930s and early 1940s, where analytical methodology did not appropriately control for post-sampling and storage-induced degradation of ascorbate. Considering the labile nature of vitC $^{(33)}$, there is reason to believe that the authors may have significantly underestimated the vitC content of the blood samples in the absence of adequate technology. Moreover, the majority of the individuals included were in fact not severely vitC deficient by current definitions ${ }^{(25)}$. Consequently, it remains to be investigated to what extent the present data can be translated to human pregnancies.

Recent data emphasise SVCT2 as the dominant contributor to the placental transfer of $\operatorname{vitC}^{(12,18,19,43)}$ and in vivo studies in SVCT2 $2^{(-/-)}$mice have strongly suggested that placental uptake of vitC requires functional SVCT2 transporters ${ }^{(43)}$. Thus, regulatory mechanisms associated with a preferential transport across the placenta are most likely to be SVCT2mediated. The present findings suggest that maternal deficiency during gestation may override such preferential maternal transport, sustaining a basal maternal vitC concentration preventing maternal scurvy at the expense of the offspring.

Natural selection favouring short-term survival over longterm health and reproduction through the allocation of scarce micronutrients by triage is a known hypothesis ${ }^{(44)}$ and has been reported, for example, for vitamin $\mathrm{K}$ and $\mathrm{Se}^{(45,46)}$. Reports have stated that vitC concentrations in fetal blood decrease towards birth $^{(47,48)}$ and up to $50 \%$ perinatally ${ }^{(49-51)}$; thus, postnatally obtained plasma samples might differ from the prenatal level. However, mean vitC concentrations in newborn CTRL pups were 50-fold higher in plasma and 3-fold higher in brain tissue compared with newborn DEF pups, making comparable fetal vitC exposure in utero highly unlikely.

VitC is well accepted to have a pivotal role as an antioxidant and in the maintenance of redox homeostasis, and serves as a cofactor in the formation and reuptake of neurotransmitters in the brain ${ }^{(8)}$. The developing brain is highly susceptible to oxidative damage due to immaturity and decreased levels and synthesis of antioxidant enzymes, high oxygen consumption and readily oxidisable PUFA ${ }^{(52)}$. Increased oxidative stress in the brain has been shown in SVCT2 $2^{(-/-)}$mice delivered by caesarian sections close to term ${ }^{(43)}$ and in the offspring of gulonolactone oxidase-knockout mice unable to synthesise vitC $^{(53)}$; thus, it is conceivable that maternal vitC deficiency leads to prenatal oxidative stress. In humans, oxidative stress has been suggested to play a role in the pathogenesis of a number of diseases in the pregnant mother and the newborn ${ }^{(54)}$, including intraventricular haemorrhage leading to neonatal brain damage ${ }^{(55)}$. Furthermore, oxidative stress has been suggested as a plausible link between adverse fetal development and pregnancy outcome and the risk of developing chronic diseases later in life ${ }^{(56)}$. However, in the present study, no differences in oxidative stress markers - apart from vitC itself - were observed between the groups, suggesting that vitC deficiency imposed on these animals did not induce oxidative stress in the narrow time window studied

Table 1. Antioxidant status and lipid oxidation markers in the brains of newborn guinea pig pups

(Mean values and standard deviations)

\begin{tabular}{|c|c|c|c|c|}
\hline & \multicolumn{2}{|c|}{ Controls } & \multicolumn{2}{|c|}{ Deficient } \\
\hline & Mean & SD & Mean & SD \\
\hline$n$ & \multicolumn{2}{|c|}{10} & \multicolumn{2}{|c|}{11} \\
\hline Vitamin C (nmol/g tissue) & 1816 & 105 & $659^{\star \star *}$ & 102 \\
\hline AOR (\% oxidised of total vitC) $\dagger$ & 4.0 & 3.6 & $6 \cdot 3$ & 4.7 \\
\hline Glutathione (nmol/g tissue) & 1268 & 67 & 1330 & 90 \\
\hline Malondialdehyde (nmol/g tissue) & 530 & 74 & 566 & 86 \\
\hline 8- $\mathrm{F}_{2}$-isoprostanes (ng/g tissue) & $8 \cdot 1$ & $1 \cdot 1$ & $8 \cdot 2$ & 1.4 \\
\hline
\end{tabular}

AOR, ascorbate oxidation ratio; vitC, vitamin $\mathrm{C}$.

${ }^{* \star *}$ Mean value was significantly different compared with the control animals $(P<0.001$; one-way ANOVA using Tukey's post hoc test of planned comparisons).

†AOR is calculated as dehydroascorbic acid divided by total vitC in percentage. 
here (Table 1), although the brain levels of vitC in the DEF newborns were decreased by approximately $60 \%$ compared with the CTRL group. This observation is in accordance with previously obtained results ${ }^{(9,10)}$ indicating that alterations in redox homeostasis may not be the primary course underlying the detrimental effects of vitC deficiency on brain development ${ }^{(9,10)}$.

Mice devoid of SVCT2 die immediately after birth, displaying intraparenchymal brain haemorrhage emphasising an essential role of vitC in sustaining perinatal survival and normal brain development ${ }^{(12)}$. This finding has been confirmed by Harrison et $a l .^{(43)}$; SVCT2 ${ }^{(-/-)}$newborns died shortly after birth; however, fewer SVCT2 $2^{(-/-)}$and SVCT2 $2^{(+/-)}$offspring (generated from $\mathrm{SVCT}^{(+/-)}$stock) than expected were recorded at delivery, indicating that a lack of vitC in utero contributes to increased fetal mortality. This is suggested to be, at least in part, due to an impairment of collagen IV synthesis leading to fragile capillaries and a subsequent increased risk of intracortical haemorrhages and subsequent death in utero or at parturition $^{(43)}$.

In human subjects, a low maternal intake of vitC has been associated with an increased risk of pregnancy-associated disorders such as pre-eclampsia ${ }^{(57)}$ and a variety of adverse pregnancy outcomes such as preterm delivery ${ }^{(58)}$ and decreased birth weight ${ }^{(59)}$. To reduce the risk of such adverse outcomes, intake of periconceptional multivitamins on a daily basis has been suggested ${ }^{(60,61)}$; however, other authors have failed to establish a positive correlation between maternal vitC intake and pregnancy outcome ${ }^{(62-66)}$. Hence, whether supplementation should be offered on a regular basis during pregnancy or rather in the case of potentially 'risk' pregnancies remains controversial. Moreover, to evaluate the true effect of vitC deficiency and supplementation, inclusion criteria and vitC status must be well defined between groups which, in human trials, have often been neglected, leading to inconclusive and divergent data ${ }^{(67)}$. Thus, as discussed above, there are presently insufficient data to conclude whether maternal vitC deficiency also leads to deficiency in the offspring of humans.

In conclusion, chronic maternal vitC intake during the second and third trimesters does not significantly influence pregnancy outcome with respect to gestational length, maternal weight gain, birth weight and perinatal survival in guinea pigs. However, the present data combined with previously reported results ${ }^{(12,43)}$ suggest that an inadequate maternal vitC intake during pregnancy may indeed pose a risk to the fetus. The findings of reduced plasma vitC in newborn DEF pups compared with their mothers indicate that preferential transport of vitC from the mother to the fetus is overridden during a prolonged maternal vitC deficiency, hereby maintaining a basal maternal vitC concentration at the expense of the offspring. The present results contradict the notion that a fetus is protected from vitC deficiency and suggest that a prolonged maternal vitC deficiency may have a detrimental effect on fetal brain development in utero. Well-designed clinical studies of the impact of maternal vitC deficiency on fetal development are therefore warranted.

\section{Acknowledgements}

The authors wish to thank Annie Bjergby Kristensen, Elisabeth Veyhe Andersen and Joan Frandsen for their excellent technical assistance. The present study was supported by grants from the Danish National Research Council (\#FSS271-080763) and The LIFEPHARM Centre for in vivo pharmacology to J. L. The authors' contributions were as follows: J. G. S., P. T.-N. and J. L. planned the study; J. G. S. and P. T.-N. conducted the in vivo experiment; J. G. S. and J. L. performed the data analysis; J. G. S., P. T.-N. and J. L. wrote the paper. The authors declare no conflict of interest which could influence the presented work.

\section{References}

1. Guesnet P \& Alessandri JM (2011) Docosahexaenoic acid (DHA) and the developing central nervous system (CNS) implications for dietary recommendations. Biochimie $\mathbf{9 3}$, 7-12.

2. Birch EE, Carlson SE, Hoffman DR, et al. (2010) The DIAMOND (DHA Intake And Measurement Of Neural Development) Study: a double-masked, randomized controlled clinical trial of the maturation of infant visual acuity as a function of the dietary level of docosahexaenoic acid. Am J Clin Nutr 91, 848-859.

3. Lozoff B (2011) Early iron deficiency has brain and behavior effects consistent with dopaminergic dysfunction. I Nutr 141, 740S-746S.

4. Roth C, Magnus P, Schjolberg S, et al. (2011) Folic acid supplements in pregnancy and severe language delay in children. JAMA 306, 1566-1573.

5. Christian P, Murray-Kolb LE, Khatry SK, et al. (2010) Prenatal micronutrient supplementation and intellectual and motor function in early school-aged children in Nepal. JAMA 304, $2716-2723$.

6. Christian P (2010) Micronutrients, birth weight, and survival. Annu Rev Nutr 30, 83-104.

7. McDonald SD, Han Z, Mulla S, et al. (2010) Overweight and obesity in mothers and risk of preterm birth and low birth weight infants: systematic review and meta-analyses. $\mathrm{Br}$ Med J 341, c3428.

8. Tveden-Nyborg P \& Lykkesfeldt J (2009) Does vitamin C deficiency result in impaired brain development in infants? Redox Rep 14, 2-6.

9. Tveden-Nyborg P, Vogt L, Schjoldager JG, et al. (2012) Maternal vitamin $\mathrm{C}$ deficiency during pregnancy persistently impairs hippocampal neurogenesis in offspring of guinea pigs. PLOS One 7, e48488.

10. Tveden-Nyborg P, Johansen LK, Raida Z, et al. (2009) Vitamin C deficiency in early postnatal life impairs spatial memory and reduces the number of hippocampal neurons in guinea pigs. Am J Clin Nutr 90, 540-546.

11. Lykkesfeldt J, Trueba GP, Poulsen HE, et al. (2007) Vitamin C deficiency in weanling guinea pigs: differential expression of oxidative stress and DNA repair in liver and brain. Br J Nutr 98, 1116-1119.

12. Sotiriou S, Gispert S, Cheng J, et al. (2002) Ascorbic-acid transporter Slc23a1 is essential for vitamin $\mathrm{C}$ transport into the brain and for perinatal survival. Nat Med 8, 514-517.

13. Frikke-Schmidt $\mathrm{H}$, Tveden-Nyborg $\mathrm{P}$, Lykkesfeldt $\mathrm{J}$, et al. (2011) Vitamin C in human nutrition. In Vitamins for Prevention of Human Diseases, 1st ed., pp. 323-347 [W Hermann 
and R Obeid, editors]. Berlin/New York: Walter de Gruyter $\mathrm{GmbH} \& \mathrm{Co}$. KG.

14. Duarte TL \& Lunec J (2005) Review: when is an antioxidant not an antioxidant? A review of novel actions and reactions of vitamin C. Free Radic Res 39, 671-686.

15. Teel HM, Burke BS \& Draper R (1938) Vitamin C in human pregnancy and lactation I studies during pregnancy. $A m \mathrm{~J}$ Dis Child 56, 1004-1010.

16. Dejmek J, Ginter E, Solansky I, et al. (2002) Vitamin C, E and A levels in maternal and fetal blood for Czech and gypsy ethnic groups in the Czech Republic. Int J Vitam Nutr Res 72, 183-190.

17. de Oliveira AM, Rondo PHD \& Barros SBD (2004) Concentrations of ascorbic acid in the plasma of pregnant smokers and nonsmokers and their newborns. Int J Vitam Nutr Res 74, 193-198.

18. Prasad PD, Huang W, Wang H, et al. (1998) Transport mechanisms for vitamin $\mathrm{C}$ in the JAR human placental choriocarcinoma cell line. Biochim Biophys Acta 1369, 141-151.

19. Biondi C, Pavan B, Dalpiaz A, et al. (2007) Expression and characterization of vitamin $\mathrm{C}$ transporter in the human trophoblast cell line HTR-8/SVneo: effect of steroids, flavonoids and NSAIDs. Mol Hum Reprod 13, 77-83.

20. Huppertz B (2008) The anatomy of the normal placenta. J Clin Pathol 61, 1296-1302.

21. Carter AM (2007) Animal models of human placentation - a review. Placenta 28, S41-S47.

22. Enders AC (1965) A comparative study of fine structure of trophoblast in several hemochorial placentas. Am J Anat 116, 29-67.

23. Frei B, Birlouez-Aragon I \& Lykkesfeldt J (2012) Authors' perspective: what is the optimum intake of vitamin $\mathrm{C}$ in humans? Crit Rev Food Sci Nutr 52, 815-829.

24. Levine M, Conry-Cantilena C, Wang YH, et al. (1996) Vitamin C pharmacokinetics in healthy volunteers: evidence for a recommended dietary allowance. Proc Natl Acad Sci U S A 93, 3704-3709.

25. Smith JL \& Hodges RE (1987) Serum levels of vitamin C in relation to dietary and supplemental intake of vitamin $\mathrm{C}$ in smokers and nonsmokers. Ann N Y Acad Sci 498, 144-152.

26. Schleicher RL, Carroll MD, Ford ES, et al. (2009) Serum vitamin $\mathrm{C}$ and the prevalence of vitamin C deficiency in the United States: 2003-2004 National Health and Nutrition Examination Survey (NHANES). Am J Clin Nutr 90, 12521263.

27. Villalpando S, Montalvo-Velarde I, Zambrano N, et al. (2003) Vitamins A, and C and folate status in Mexican children under 12 years and women 12-49 years: a probabilistic national survey. Salud Publica Mex 45, S508-S519.

28. Lykkesfeldt J (2006) Smoking depletes vitamin C: should smokers be recommended to take supplements? In Cigarette Smoke \& Oxidative Stress, pp. 237-260 [B Halliwell and HE Poulsen, editors]. Berlin: Springer.

29. Lykkesfeldt J \& Moos T (2005) Age-dependent change in vitamin $C$ status: a phenomenon of maturation rather than of ageing. Mech Ageing Dev 126, 892-898.

30. Tveden-Nyborg P, Hasselholt S, Miyashita N, et al. (2012) Chronic vitamin $\mathrm{C}$ deficiency does not accelerate oxidative stress in ageing brains of guinea pigs. Basic Clin Pharmacol Toxicol 110, 524-529.

31. Lykkesfeldt J (2012) Ascorbate and dehydroascorbic acid as biomarkers of oxidative stress: validity of clinical data depends on vacutainer system used. Nutr Res 32, 66-69.

32. Lykkesfeldt J (2000) Determination of ascorbic acid and dehydroascorbic acid in biological samples by high-performance liquid chromatography using subtraction methods: reliable reduction with tris[2-carboxyethyl] phosphine hydrochloride. Anal Biochem 282, 89-93.

33. Lykkesfeldt J (2007) Ascorbate and dehydroascorbic acid as reliable biomarkers of oxidative stress: analytical reproducibility and long-term stability of plasma samples subjected to acidic deproteinization. Cancer Epidemiol Biomarkers Prev 16, 2513-2516

34. Hissin PJ \& Hilf R (1976) A fluorometric method for determination of oxidized and reduced glutathione in tissues. Anal Biochem 74, 214-226.

35. Lykkesfeldt J (2001) Determination of malondialdehyde as dithiobarbituric acid adduct in biological samples by HPLC with fluorescence detection: comparison with ultraviolet-visible spectrophotometry. Clin Chem $\mathbf{4 7}$, $1725-1727$.

36. Pate SK, Lukert BP \& Kipp DE (1996) Tissue vitamin C levels of guinea pig offspring are influenced by maternal vitamin $\mathrm{C}$ intake during pregnancy. J Nutr Biochem 7, 524-528.

37. Wegger I \& Palludan B (1994) Vitamin C deficiency causes hematological and skeletal abnormalities during fetal development in swine. J Nutr 124, 241-248.

38. Bates CJ, Cowen TD \& Tsuchiya H (1988) Growth, ascorbic acid and iron contents of tissues of young guineapigs whose dams received high or low levels of dietary ascorbic acid or Fe during pregnancy and suckling. $\mathrm{Br} J$ Nutr 60, 487-497.

39. Norkus EP, Bassi J \& Rosso P (1979) Maternal-fetal transfer of ascorbic acid in the guinea pig. J Nutr 109, 2205-2212.

40. Haig D (1993) Genetic conflicts in human pregnancy. Q Rev Biol 68, 495-532.

41. Haig D (2004) Evolutionary conflicts in pregnancy and calcium metabolism - a review. Placenta 25, S10-S15.

42. Lund CJ \& Kimble MS (1943) Some determinants of maternal and plasma vitamin C levels. Am J Obstet Gynecol 46, 635-647.

43. Harrison FE, Dawes SM, Meredith ME, et al. (2010) Low vitamin $\mathrm{C}$ and increased oxidative stress and cell death in mice that lack the sodium-dependent vitamin $\mathrm{C}$ transporter SVCT2. Free Radic Biol Med 49, 821-829.

44. Ames BN (2006) Low micronutrient intake may accelerate the degenerative diseases of aging through allocation of scarce micronutrients by triage. Proc Natl Acad Sci US A 103, 17589-17594.

45. Mccann JC \& Ames BN (2009) Vitamin K, an example of triage theory: is micronutrient inadequacy linked to diseases of aging? Am J Clin Nutr 90, 889-907.

46. Mccann JC \& Ames BN (2011) Adaptive dysfunction of selenoproteins from the perspective of the triage theory: why modest selenium deficiency may increase risk of diseases of aging. FASEB J 25, 1793-1814.

47. Zalani S, Rajalakshmi R \& Parekh LJ (1989) Ascorbic acid concentration of human fetal tissues in relation to fetal size and gestational age. Br J Nutr 61, 601-606.

48. Das S \& Powers HJ (1998) The effects of maternal intake and gestational age on materno-fetal transport of vitamin $\mathrm{C}$ in the guinea-pig. Br J Nutr 80, 485-491.

49. Braestrup PW (1938) The content of reduced ascorbic acid in blood plasma in infants, especially at birth and in the first days of life. J Nutr 16, 363-373.

50. Hamil BM, Munks B, Moyer EZ, et al. (1947) Vitamin C in the blood and urine of the newborn and in the cord and maternal blood. Am J Dis Child 74, 417-433.

51. Norkus EP \& Rosso P (1981) Effects of maternal intake of ascorbic acid on the postnatal metabolism of this vitamin in the guinea pig. J Nutr 111, 624-630. 
52. Ikonomidou C \& Kaindl AM (2011) Neuronal death and oxidative stress in the developing brain. Antioxid Redox Sign 14, 1535-1550.

53. Harrison FE, Meredith ME, Dawes SM, et al. (2010) Low ascorbic acid and increased oxidative stress in gulo $(-/-)$ mice during development. Brain Res 1349, 143-152.

54. Gitto E, Reiter RJ, Karbownik M, et al. (2002) Causes of oxidative stress in the pre- and perinatal period. Biol Neonate 81, 146-157.

55. Buonocore G, Perrone S, Longini M, et al. (2003) Non protein bound iron as early predictive marker of neonatal brain damage. Brain 126, 1224-1230.

56. Luo ZC, Fraser WD, Julien P, et al. (2006) Tracing the origins of "fetal origins" of adult diseases: programming by oxidative stress? Med Hypotheses 66, 38-44.

57. Zhang C, Williams MA, King IB, et al. (2002) Vitamin C and the risk of preeclampsia - results from dietary questionnaire and plasma assay. Epidemiology 13, 409-416.

58. Siega-Riz AM, Promislow JHE, Savitz DA, et al. (2003) Vitamin $\mathrm{C}$ intake and the risk of preterm delivery. $A m \mathrm{~J}$ Obstet Gynecol 189, 519-525.

59. Lee BE, Hong YC, Lee KH, et al. (2004) Influence of maternal serum levels of vitamins $\mathrm{C}$ and $\mathrm{E}$ during the second trimester on birth weight and length. Eur J Clin Nutr 58, $1365-1371$.
60. Catov JM, Bodnar LM, Olsen J, et al. (2011) Periconceptional multivitamin use and risk of preterm or small-for-gestationalage births in the Danish National Birth Cohort. Am J Clin Nutr 94, 906-912.

61. Hasan R, Olshan AF, Herring AH, et al. (2009) Self-reported vitamin supplementation in early pregnancy and risk of miscarriage. Am J Epidemiol 169, 1312-1318.

62. Roberts JM, Myatt L, Spong CY, et al. (2010) Vitamins C and E to prevent complications of pregnancy-associated hypertension. $N$ Engl J Med 362, 1282-1291.

63. Hauth JC, Clifton RG, Roberts JM, et al. (2010) Vitamin C and E supplementation to prevent spontaneous preterm birth a randomized controlled trial. Obstet Gynecol 116, 653-658.

64. Spinnato JA, Freire S, Silva JLPE, et al. (2007) Antioxidant therapy to prevent preeclampsia - a randomized controlled trial. Obstet Gynecol 110, 1311-1318.

65. Rumbold A, Middleton P, Pan N, et al. (2011) Vitamin supplementation for preventing miscarriage. The Cochrane Database of Systematic Reviews, CD004073.

66. Wang YZ, Ren WH, Liao WQ, et al. (2009) Concentrations of antioxidant vitamins in maternal and cord serum and their effect on birth outcomes. J Nutr Sci Vitaminol (Tokyo) 55, 1-8.

67. Lykkesfeldt J \& Poulsen HE (2010) Is vitamin C supplementation beneficial? Lessons learned from randomised controlled trials. Br J Nutr 103, 1251-1259. 